\title{
Rectification of RF Fields in Load Dependent Coupled Systems: Application to Non-Invasive Electroceuticals
}

\author{
Sree N. Koneru ${ }^{1}$, Charles R. Westgate ${ }^{2}$, Kenneth J. McLeod ${ }^{1}$ \\ ${ }^{1}$ Clinical Science and Engineering Research Laboratory, Binghamton University, Binghamton, NY, USA \\ ${ }^{2}$ Department of Electrical and Computer Engineering, Binghamton University, Binghamton, NY, USA \\ Email: "skoneru1@binghamton.edu
}

Received 21 August 2015; accepted 26 February 2016; published 29 February 2016

Copyright (C) 2016 by authors and Scientific Research Publishing Inc.

This work is licensed under the Creative Commons Attribution International License (CC BY).

http://creativecommons.org/licenses/by/4.0/

(c) (i) Open Access

\begin{abstract}
Electroceuticals are medical devices that employ electric signals to alter the activity of specific nerve fibers to achieve therapeutic effects. The rapid growth of RF microelectronics has resulted in the development of very small, portable, and inexpensive shortwave and microwave radio frequency (RF) amplifiers, raising the possibility of utilizing these new RF technologies to develop non-contact electroceutical devices. However, the bio-electromagnetics literature suggests that beyond $10 \mathrm{MHz}$, RF fields cannot influence biological tissue, beyond simple heating, because effective demodulation mechanisms at these frequencies do not exist in the body. However, RF amplifiers operating at or near saturation have non-linear interactions with complex loads, and if body tissue creates a complex loading condition, the opportunity exists for the coupled system to produce non-linear effects, that is, the equivalent of demodulation may occur. Correspondingly, exposure of tissue to pulsed RF energy could result in the creation of low frequency demodulation components capable of influencing tissue activity. Here, we develop a one-dimensional, numerical simulation to investigate the complex loading conditions under which such demodulation could arise. Applying these results in a physical prototype device, we show that up to $7.5 \%$ demodulation can be obtained for a $40 \mathrm{MHz}$ RF field pulsed at $1 \mathrm{KHz}$. Implications for this research include the possibility of developing wearable, electromagnetic electroceutical devices.
\end{abstract}

\section{Keywords}

RF Demodulation in Tissue, Non-Linear Coupling, Complex Load-Line, Electroceuticals, Neuromodulation, Stochastic Resonance

\footnotetext{
${ }^{*}$ Corresponding author.
}

How to cite this paper: Koneru, S.N., Westgate, C.R. and McLeod, K.J. (2016) Rectification of RF Fields in Load Dependent Coupled Systems: Application to Non-Invasive Electroceuticals. J. Biomedical Science and Engineering, 9, 112-121. 


\section{Introduction}

Electroceuticals are bioelectronic devices which rely on electrical fields induced into tissues to influence neural activity and thereby achieve therapeutic effects [1]. While the term "electroceuticals" was coined only recently in light of renewed efforts to expand research in the area, electric fields have been used in medicine for the larger part of the $20^{\text {th }}$ century. Diathermy devices have existed since the 1920 's to treat a broad range of health conditions from inflammation to tumors; defibrillators were developed in the late 1930's; and, pacemakers, muscle stimulators, transcutaneous nerve stimulators (TENS), spinal cord stimulators and most recently, deep brain stimulators, have subsequently been introduced into medicine. A critical development in the field of electroceuticals has been the transition from reliance on relatively high intensity $(1-100 \mathrm{~V} / \mathrm{m})$, supra-threshold, electric fields which are sufficient to deterministically excite the neuromuscular system (i.e. neurostimulation) [2] to neuromodulation approaches, wherein the induced electric fields are sub-threshold [3]. Neuromodulation approaches do not ensure initiation of action potentials, but are sufficient to bias neural activity. Nonetheless, most of the current neuromodulatory technologies being developed still rely on skin contact or other invasive means to deliver electrical currents into the tissue. As a result, even when using the lower level, high frequency stimuli, skin irritation or damage can arise from chronic transcutaneous neurostimulation. Further, surgical risks involved in invasive neurostimulation techniques and the need for carefully monitored use have slowed the development and widespread use of electroceuticals in medicine.

The need for much smaller induced electric field intensities in neuromodulation processes, as compared to neurostimulation, creates an opportunity to pursue alternative field induction mechanisms. Specifically, the ability of magnetic fields (EMF) to readily penetrate clothing, bandages, and skin, raises the possibility of developing "contactless" electroceuticals. The magnitude of the electric field induced in tissue is directly proportional to the magnitude and frequency of an applied magnetic field, and so high frequency, that is, radio-frequency (RF) fields would seem to have certain advantages in this application as RF based devices would not require large magnetic field intensities. However, while RF fields readily penetrate into the body, physiologic processes are responsive only to relatively low frequencies, typically in the $\mathrm{KHz}$ range or lower. Therefore, in order to influence the electro-physiologic behavior of tissue with RF fields, it is necessary to modulate the RF waveform and then ensure the RF waveform is demodulated within the tissue. Such demodulation requires a non-linear or rectification process within the tissue.

At frequencies below $10 \mathrm{MHz}$ electric fields induced into tissues are largely excluded from the interior of cells due to the low conductivity of the cell plasma membrane. The charge build-up on the plasma membraneperturbs the normal transmembrane potential and can influence the gating of the non-linear ion channels present in the cell membrane [4]. That is, for fields below $10 \mathrm{MHz}$, the cell membrane can, in principle, demodulate alternating electric fields in the tissue. Alternatively, electric fields higher than about $10 \mathrm{MHz}$ pass through the cell as a result of the low capacitive reactance of the membrane at these higher frequencies. Consistent with this biophysical premise, experimentation has confirmed that for frequencies greater than $10 \mathrm{MHz}$, the non-linear rectifying behavior of the cell membrane is insufficient to initiate any significant biological effects at field intensities below those which create significant heating, and to date, no other non-linear processes in tissues has been identified which could serve to demodulate RF fields over $10 \mathrm{MHz}$ [5].

However, when low-power RF transmitters are operated in close proximity to the body, a very different exposure condition exists. Specifically, to maximize efficiency, RF amplifiers are typically operated near saturation, but operation near saturation increases system non-linearity [6]. This non-linearity is strongly evident when the load is complex, resulting in the operational "load-line" contorting into an ellipse at high output levels (Figure 1) (Salib, et al., 1987). This behavior is commonly observed in commercially available RF power amplifiers (Figure 2). The net result is that while the load (i.e. tissue) may be linear, the coupled RF system may behave similarly to an ideal electric field generator driving a non-linear load. We hypothesize that this load-amplifier interaction could result in significant RF field demodulation in a living system. We approach this question in two parts: First, we investigate whether "demodulation" can be demonstrated under complex load conditions, using a numerical simulation approach. Second, using a low power RF amplifier operating near saturation, we test the predictions made by the numerical simulations. With this prototype system, we were able to demonstrate a $7.5 \%$ demodulation efficiency, confirming the plausibility of such effects occurring in vivo. 


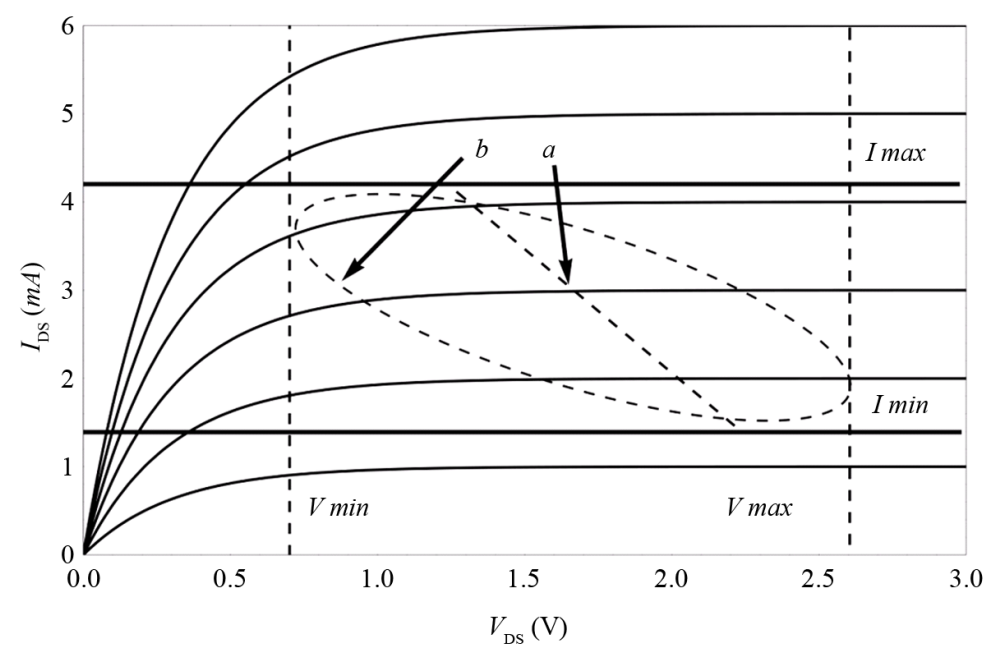

Figure 1. Load-lines, representing the trajectory of all possible instantaneous values of current $\left(I_{\mathrm{DS}}\right)$ and voltage $\left(V_{\mathrm{DS}}\right)$, for a typical electronic amplifying device operating under two loading conditions: (a) small signal, resistive loading; (b) large signal (operation in or near saturation) with a complex load resulting in an elliptical load line.

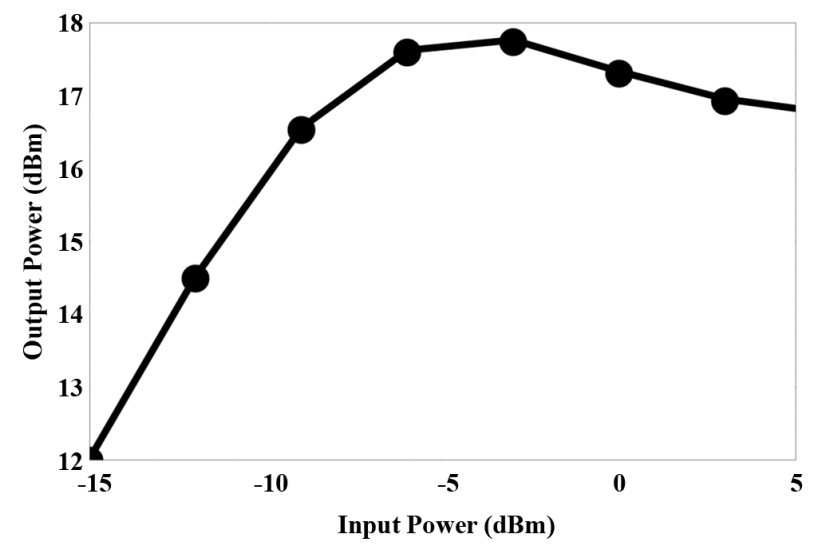

Figure 2. Output power measurements from a $150 \mathrm{~mW}$ wideband (10 MHz-3 GHz) power amplifier (RF-Lambda Model RAMP01M03GC), driven at $915 \mathrm{MHz}$ (Wavetek Model 1080) and terminated into a loop antenna. The output response when approaching and reaching saturation is typical of RF power amplifiers where a decrease in output power occurs for increased input power.

\section{Methods}

\subsection{Part $1-$ Numerical Simulations}

We initially tested whether demodulation can be demonstrated under coupled loading conditions, by employing numerical simulation. More specifically, we investigated if demodulation can occur when a weak RF amplifier operating near saturation is coupled into complex loads. At frequencies above $10 \mathrm{MHz}$, human tissue appears to be strictly resistive, that is, it presents a linear load. However, human tissues tend to be physically large relative to the wavelengths of interest. For example, at $100 \mathrm{MHz}$, the dielectric permittivity of tissue is essentially the permittivity of water, or close to $100 \mathrm{~F} / \mathrm{m}$. Correspondingly, the wavelength of $100 \mathrm{MHz}$ in body tissue will be around $30 \mathrm{~cm}$. Therefore, while for such frequencies there are no explicit capacitive or inductive reactance elements in human tissue, the time delays inherent to wave transmission in the body gives rise to wave behavior as if physical capacitive or inductive load elements were present.

Analysis of RF amplifier systems, driven to near saturation, with complex loading, generally precludes numerical analysis, and so developers commonly rely on "load-pull" evaluation techniques when developing such 
systems [7]. Load-pull is an empirical technique wherein the load is altered and the output of the amplifier is directly measured. Here, to permit numerical analysis, we utilized a much simplified one-dimensional model (Figure 3). The model incorporates an RF oscillator/amplifier driving a loop antenna as the field source. The exposed tissue is represented as a pick-up coil with impedance $R_{P}$. To introduce the necessary time delays associated with the large physical structure of human tissues, we utilized a transmission line with length on the order of a fraction of the wavelength of interest. The transmission line, of impedance $Z_{T}$, terminates with an impedance $Z_{L}$, which may be matched or unmatched to that of the transmission line. This terminating impedance may reflect, for example, that of bone, while the bulk of the tissue (transmission line) would have the impedance of muscle, thereby creating an unmatched load condition.

The electric field existing at the exposed tissue site, i.e. across $R_{P}$, is evaluated through a time series simulation as the sum of the incident and reflected waves in the system. The amplitude of the reflected wave is calculated as [8]:

$$
V_{R}(\omega)=V_{I}(\omega) \times \Gamma(\omega)
$$

where:

$$
\begin{aligned}
& V_{R}=\text { Intensity of reflected wave } \\
& \omega=\text { Oscillator frequency } \\
& V_{I}=\text { Intensity of incident wave } \\
& \Gamma=\text { Reflection coefficient }=\left(Z_{L}-Z_{T}\right) /\left(Z_{L}+Z_{T}\right)
\end{aligned}
$$

We evaluated system behavior under conditions of both small-signal and large-signal amplifier operation into a complex load, the former using linear I-V load line characteristics, and the latter using elliptical load line characteristics. For the latter analyses, we approximated the load line as:

$$
V_{I}=a \times \mathrm{e}^{\frac{-\left(I_{p}-b\right)^{2}}{c}}
$$

where $I_{P}=$ Current in pick-up coil; and parameters $a, b \& c$ represent the height, position and width of the elliptical load-line portion respectively.

\section{Numerical Model Algorithm}

The modeling algorithm involved an eight step process which tracked each half-cycle of the oscillator output as it traversed the model system of Figure 4:

1) Production of an RF current (and corresponding magnetic field) in the loop antenna.

2) Induction of an RF current in the pick-up coil.

3) The development of an electric potential across the resistive load in the pick-up coil that includes $R_{p}$ in parallel with the transmission line input impedance.

4) Transmission of an incident traveling half-cycle down the transmission line.

5) Reflection of the travelling half-cycle at the terminating load.

6) Transmission of the reflected half-cycle back towards the pick-up coil.
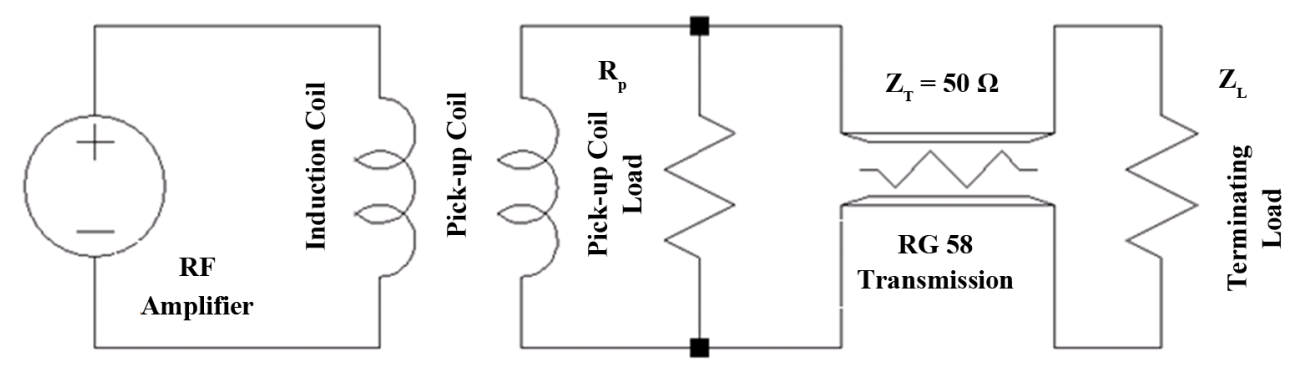

Figure 3. Schematic of the transmitter-tissue model. The transmitter and complex load (representing body tissue) are inductively coupled to each other. The layers of tissue (thickness) are represented by the length of a lossy transmission line. The RF amplifier induces a field in a pick-up coil (first layer of tissue) which is coupled to a terminating load andmeasured via a transmission line, where the transmission line represents layers of tissue. The terminating load is varied, representing, for example, the impedance of bone or air, resulting in a range of reflective coefficients. 

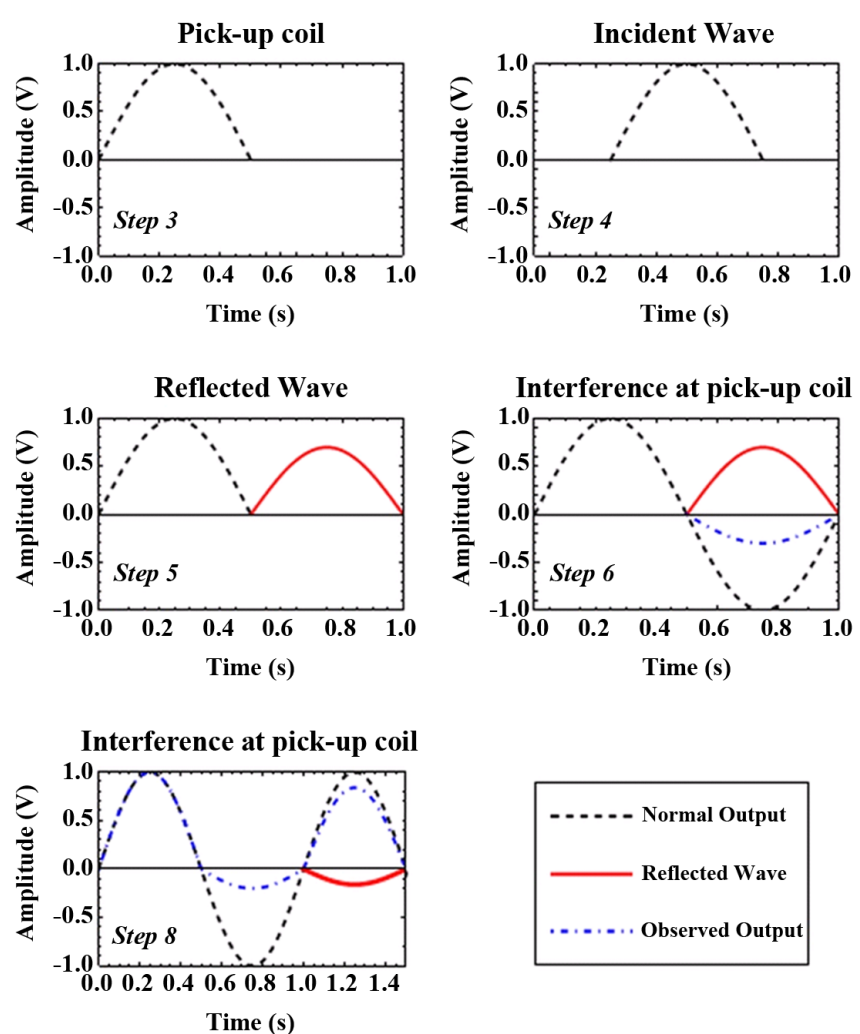

Figure 4. Results at each time step of the simulation model performed for the conditions of $\Gamma=0.7$, transmission line length $=1 / 4 \lambda$. Steps 3 through 6 follow the sequence of the first $1 / 2$ wave cycle induced into the pick-up coil. Step 8 shows the beginning of the second half wave with its reduced amplitude.

7) Development of a feedback current in the pick-up coil with the corresponding creation of a back-EMF.

8) Determination of the amplifier output current under the combined loading of the pick-up coil load current and the back EMF

100 points per half-cycle were simulated to ensure a smooth representation of the waveforms, and the simulation was continued until a steady state in the output voltage was observed. For all simulations, the D.C. component of the output voltage across the pick-up coil load $\left(R_{P}\right)$ was calculated by averaging over each complete cycle for the duration of simulation period. Simulations for various transmission line lengths (i.e. time delays) and reflection coefficients were performed to investigate the influence of the reflected wave components on the potential recorded across the pick-up coil load $\left(R_{P}\right)$.

\subsection{Part 2-Physical Measurements}

We also tested whether demodulation under complex loading of a saturated RF amplifier could be demonstrated in a physical model. A prototype RF oscillator device was built to verify the outcomes predicted by the numerical model. The physical model consisted of: 1) A battery operated $40 \mathrm{MHz}$ Colpitts-type RF oscillator with a 10 $\mathrm{cm}$ circular loop antenna. A timing circuit pulsed the oscillator at $1 \mathrm{KHz}$ utilizing a $10 \%$ duty cycle. 2) A $10 \mathrm{~cm}$ circular pick-up coil with a $500 \Omega$ resistive load, represented a human tissue load 3) RG 58 co-axialcable (50 $\Omega$; velocity factor 0.67 ) of a fixed length was used to represent a thick layer of muscle 4) An oscilloscope (Agilent Technologies, model MSO7104B) was used to measure electric potential as well as to present a terminating resistive load $\left(Z_{L}\right)$ of either $50 \Omega$ or $1 \mathrm{M} \Omega$, in parallel with $15 \mathrm{pF}$.

The two parameters varied in the testing of the physical model were electrical length of the co-axial cable and terminating impedance. Four different lengths of RG-58 co-axial cables, representing electrical lengths (at 40 $\mathrm{MHz}$ ) of $1 / 4 \lambda, 1 / 2 \lambda, 3 / 4 \lambda$ and $1 \lambda$ were used. Recordings were obtained with both terminating load conditions, resulting in eight possible measurement combinations using the two parameters. 


\section{Results}

\subsection{Numerical Results-Small Signal Operation}

Simulation results under conditions for which the amplifier output was not load dependent (i.e. small signal, non-saturated amplifier operation), using a $1 / 4 \lambda$ transmission line length with a terminating load condition of $\Gamma$ $=0.7$ illustrates the overall behavior of the system (Figure 5(a)). The initial half-cycle induced in the pick-up coil travels down the transmission line and is reflected back with the same sign as the incident half-cycle, but at reduced amplitude. The reflected wave arrives back at the pick-up coil as the amplifier output is changing polarity. For a load independent oscillator, the output of the oscillator is no different than that expected in the absence of a reflected wave, and so the voltage across $R_{P}$ due to the reflected wave and that due to the generator's output simply add. This results in reduced amplitude of the subsequent half-wave, which in turn proceeds down the transmission line. Correspondingly, the average value of the potential across the $\mathrm{R}_{\mathrm{P}}$ will have a net $\mathrm{DC}$ component when averaged over a full cycle of the oscillator output with this DC component decaying over time.

Simulation results were obtained for reflection coefficients ranging from 0.6 - 0.9 (Figure 5(b)). The magnitude of the reflection coefficient is seen to have a significant effect on the duration of transient response, that is, the period of time for which a D.C. offset occurs. However, at the operating frequencies of interest (>10 MHz) this corresponds to transient time of less than 25 microseconds.

\subsection{Numerical Results-Large Signal Operation}

When an RF amplifier is operating near saturation into a complex load, the output of the device becomes load dependent (i.e. large signal) in a non-trivial manner. We modeled this load dependence per Equation 3 using values of $a=1.5, \mathrm{~b}=0.5$, and $c=1$ such that increased current demand on the amplifier results in an initial rise in output voltage. This behavior results in a positive feedback such that when the reflected wave increases the load on the output amplifier, the output of the oscillator increases. The positive feedback allows the system to attain a steady state with a net D.C. offset (Figure 6). We simulated operation of the system under a range of reflection coefficients $\Gamma=0.6-0.9$ and for transmission line lengths ranging from $0-0.5 \lambda$ (Figure 7). Rectifi-
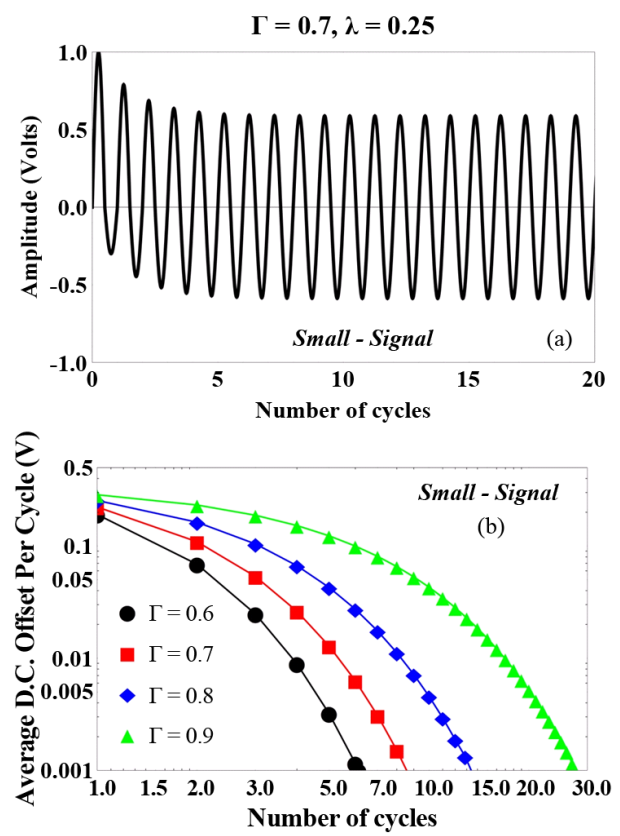

Figure 5. (a) Output at the pick-up coil load $\left(R_{P}\right)$ over 20 cycles. The waveform has the highest magnitude DC component in the first cycle which then decays rapidly over time, approaching zero after about 5 cycles. (b) D.C Offset calculated for various reflection coefficients. Higher reflection coefficients result in a slower decay of the DC component,but even at a reflection coefficient of 0.9 , the DC component decays to zero in fewer than 20 cycles. 

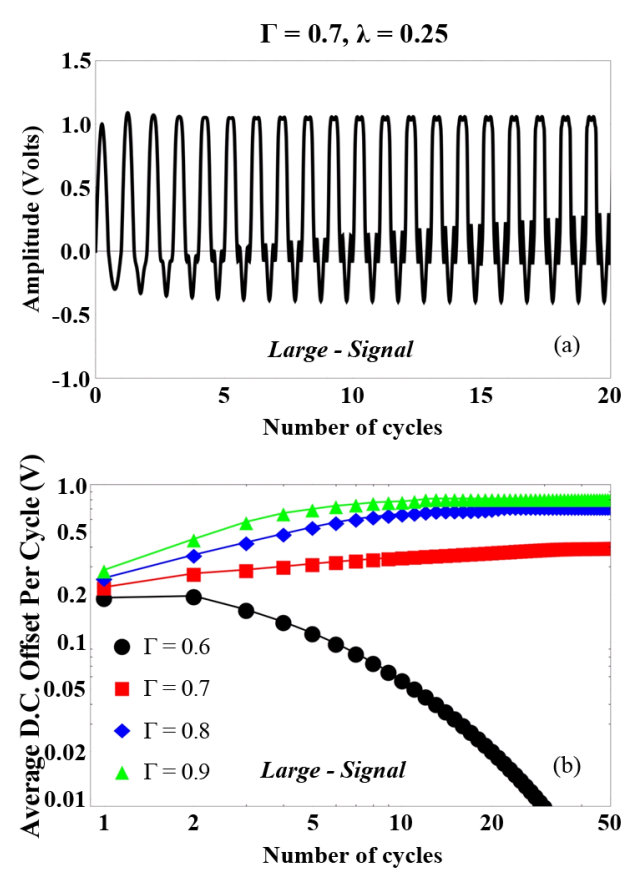

Figure 6. (a) Simulation using an elliptical load line. Output across the pick-up coil load stabilizes after an initial start-up transient with a positive peak that is much higher than the negative peak. (b) The maximum D.C. offset is about $0.6 \mathrm{~V}$ and a steady state is reached with that amplitude after approximately 20 cycles.

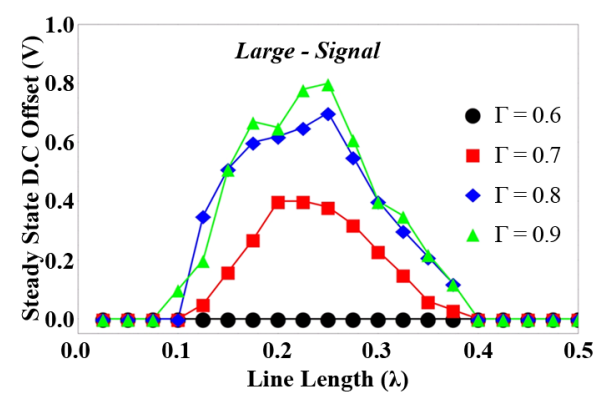

Figure 7. Steady state D.C offset amplitude for various reflection coefficients as a function of the total delay time (in units of wavelength). A reflection coeffecient of 0.9 results in amaximum DC offset (rectification) of $80 \%$. The optimaltotal delay time with respect to providing the maximum DC offset is 0.5 $\lambda$, but a rectification effect is observed over delay times of $0.3 \lambda$ to $0.7 \lambda$.

cation factors of up to $80 \%$ were obtained for reflection coefficients of 0.9 and time delays of $1 / 4$ wavelengths, but a wide range of parameter values (reflection coefficients and time delays) were found to produce significant rectification factors.

\subsection{Laboratory Model Observations}

Measurements made with the prototype $40 \mathrm{MHz}$ RF oscillator/amplifier circuit, driving a transmission line of fixed length showed distortions in the measured potential across the terminating load, resulting in a distinct D.C. offset. However, this was observed only when there was an impedance mismatch between the transmission line and terminating load. When the transmission lines were terminated with a $50 \Omega, 15 \mathrm{pF}(Z=42 \Omega, \Gamma=-0.09)$ load, the resultant waveform lacked any discernable distortion. As such, distorted waveforms were observed for only half the conditions, i.e. when the transmission lines were terminated into a $1 \mathrm{M} \Omega$ resistive load in parallel with $15 \mathrm{pF}(Z=265 \Omega ; \Gamma=0.68)$. Figure 8 shows a representative waveform when a $1 / 4 \lambda$ transmission line 


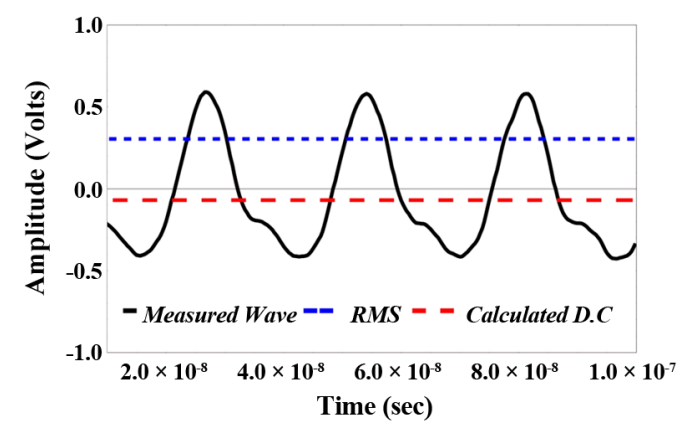

Figure 8. Waveform observed across the pick-up coil load of the prototype device when the coil load $\left(R_{P}\right)$ is linked to a unmatched terminating loadthrough a $1 / 4 \lambda$ transmission line. The waveform has peak positive and peak negative voltages of 0.6 and 0.4 respectively, with an RMS value of $0.33 \mathrm{~V}$. The D.C. component is calculated to be $0.025 \mathrm{~V}$ representing about a $7.5 \%$ demodulation efficiency.

was mismatched with the terminating load, the condition under which a maximum D.C. offset was observed. Even small deviations from the $1 / 4 \lambda$ delay significantly suppressed the D.C. offset to non-discernable levels.

\section{Discussion}

It is well known that demodulation of RF electromagnetic fields in living tissue cannot occur, at frequencies greater than $10 \mathrm{MHz}$, leading to the suggestion that high frequency stimulation should not be biologically effective. Nonetheless, a remarkably large number of experimental papers have reported biological effects associated with the use of pulsed RF stimulation. In this paper, we hypothesized that a form of demondulation could occur under certain complex loading conditions, and tested this hypothesist using both numerical and physical models.

Numerical simulation indicated that that a D.C. offset can be sustained in tissue when an RF transmitter operating at saturation, is driven into a complex load. The offset in the generated waveforms was evident in its distorted shape and was verified through numerical integration. The interference between the incident and reflected waveforms results in a time varying load being presented to the amplifier. This results in a synchronous time varying output of the amplifier, resulting in a "D.C. shift" in the output. If this RF field were modulated, for example, presented a short burst, which a relaxation time period, the field observed at the load would include a frequency component at the modulation frequency, as if demodulation had occurred. A range of reflection co-efficients $(\Gamma=0.6-0.9)$ and transmission line lengths $(0-0.5 \lambda)$ were investiated, with the best demodulation efficiency predicted for $\lambda=1 / 4$ and $\Gamma=0.9$.

Irrespective of the line length used, the physical model presented a $\Gamma$ of 0.68 when the transmission line terminated into the $1 \mathrm{M} \Omega, 15 \mathrm{pF}$ load. Consistent with the predictions made by the numerical model, the physical model showed significant distortion in the recorded waveforms and a net D.C. offset. Moreover, the best demodulation efficiency was observed when a line length of $1 / 4 \lambda$ was used, as predicted by the numerical modeling. The results from both the numerical and physical model lend support to our hypothesis that under complex loading conditions, an RF amplifier, operating near saturation, and closely coupled to the load, can produce a sustained D.C. offset in biological tissue. Correspondingly, if the RF output is modulated, energy at the modulation frequency will be observed in the tissue, even in the absence of any explicit non-linear (rectifying) component in the tissue.

A system is said to be coupled if some physical process allows components of the sytem to influence each other [9]. Consideration of the amplifier and tissue as closely coupled components as opposed to modeling the system as if the transmitter were independently acting upon the tissue (i.e. a cause-effect type relationship) is the key to our observations. In simple systems, the interactions are generally linear such that an effect is directly proportional to the causal factor. In complex systems, the interactions are, by definition, non-linear. Such systems typically give rise to emergent behaviors, that is, behaviors which cannot be predicted by evaluation of the system components in isolation. Here, the reflecting load, combined with time delays in the tissue model and the load dependent behavior inherent to low power RF amplifiers, introduces sufficient non-linear coupling that a D.C. component in the output emerges. If a more powerful amplifier were utilized such that it did not need to 
operate near saturation (large signal mode), or the tissue load was more remote from the source such that there was less feedback coupling, this non-linear pheneomenon would not be observed. Of course, the design goal in portable devices is to maximize battery lifetime, and to do so in any RF device requires operation near saturation.

While neurostimulation typically requires DC field intensities on the order $10 \mathrm{~V} / \mathrm{m}$ to be present for a duration of about 50 - 500 usec [10], neuromodulation technologies utilize far lower field intensities. In classical systems theory, random fluctuations in signals are referred to as "noise" and are nearly always considered detrimental, and so great efforts are made to eliminate this noise. However, in physiological systems, such as neural systems, while "noise" is ubiquitous, it is, in fact, widely considered to be essential in facilitating information processing in the body [11]. Stochastic Resonance (SR) is a phenomenon where the presence of "noise" in non-linear systems can be used to enhance the detection of sub-threshold stimuli. For example, in a situation where there is a detection threshold, such as exists for nerves, a subthreshold "signal” in the presence of noise may randomly exceed the necessary threshold to activate the nerve. A characteristic feature of SR in a system is that the system output, when plotted against system noise power, will produce a peak response at a non-zero value [12].

\section{Conclusion}

In summary, employing weak RF amplifiers, operating near saturation, in close proximity to the body presents the possibility of creatingnon-deterministic neuromodulation effects resulting from the development of small fields $(<10 \mathrm{~V} / \mathrm{m})$ at the frequency of RF modulation, which combine with inherent system noise in a stochastic manner (observed as a stochastic resonance). Irrespective of the type of modality, if nerve activity can be altered using modulated RF field exposure, we envision several important applications. Specifically, a popular nonpharmacological neurostimulation therapy for treating chronic pain is neural masking using Transcutaneous Electrical Nerve Stimulation [13]. However, use of this technology is limited given the risk of skin burning, and the inability to use TENS through bandages or clothing. Sub-threshold neuromodulation therapy, whether used transcutaneously or invasively, has already shown its potential in modulating a variety of nerves: the occipital nerve for treating migraines [14], sacral nerve for treating bowel incontinence [3] and vagal sympathetic complex to treat heart failure [15]. Correspondingly, the ability to modulate nerve activity without invasive procedures/skin contact would have significant advantages for those capable of benefiting from traditional neurostimulation therapies. We believe that RF electroceutical technologies, based on the principles discussed here, hold promise for the development of wearable, non-invasive devices capable of modulating larger nerve fibers and nerve plexi such as the brachial nerve plexus and sacral nerve plexus, among many others.

\section{Support}

This work was supported by BioElectronics Corporation, Frederick, MD.

\section{References}

[1] Famm, K. (2013) A Jump Start for Electroceuticals. Nature, 159-161. http://dx.doi.org/10.1038/496159a

[2] Markand, O.N., Kincaid, J.C., Pourmand, R.A., Moorthy, S.S., King, R.D., Mahomed, Y. and Brown, J.W. (1984) Electrophysiologic Evaluation of Diaphragm by Transcutaneous Phrenic Nerve Stimulation. Neurology, 604. http://dx.doi.org/10.1212/WNL.34.5.604

[3] Duelund-Jakobsen, J.E.A. (2013) Sacral Nerve Stimulation at Subsensory Threshold Does Not Compromise Treatment Efficacy: Results from a Randomized, Blinded Crossover Study. Annals of Surgery, 219-223. http://dx.doi.org/10.1097/SLA.0b013e318269d493

[4] McLeod, K.J., Rubin, C.T. and Donahue, H.J. (1995) Electromagnetic Fields in Bone Repair and Adaptation. Radio Science, 233-244. http://dx.doi.org/10.1029/94RS00832

[5] Sheppard, A.R. (2008) Quantitative Evaluations of Mechanisms of Radiofrequency Interactions with Biological Molecules and Processes. Health Physics, 95, 365-396. http://dx.doi.org/10.1097/01.HP.0000319903.20660.37

[6] Maas, S. (2003) Non-Linear Microwave and RF Circuits. 2nd Edition, Artech House, Norwood.

[7] Ghannouchi, F.M. and Hashmi, M.S. (2013) Load Pull Techniques with Application to Power Amplifier Design. Springer. http://dx.doi.org/10.1007/978-94-007-4461-5

[8] Sadiku, M.N. (2010) Electromagnetic Wave Propagation. Elements of Electromagnetics, 500-501. 
[9] Strogatz, S. (2003) Preface. Sync, Hyperion Books, 3.

[10] Mogyoros, I., Kiernan, M.C. and Burke, D. (1996) Strength-Duration Properties of Human Peripheral Nerve. Brain, 439-447. http://dx.doi.org/10.1093/brain/119.2.439

[11] McDonnell, M.D. and Ward, L.M. (2011) The Benefits of Noise in Neural Systems: Bridging Theory and Experiment. Nature Reviews Neuroscience, 415-426. http://dx.doi.org/10.1038/nrn3061

[12] McDonnell, M. and Abott, D. (2009) What Is Stochastic Resonance? Definitions, Misconceptions, Debates, and Its Relebance to Biology. PLoS Computational Biology, 5, 1-9. http://dx.doi.org/10.1371/journal.pcbi.1000348

[13] Nnoaham, K. and Kumbang, J. (2010) Transcutaneous Electrical Nerve Stimulation (TENS) for Chronic Pain. The Cochrane Collaboration, 1-62.

[14] Slotty, P.J.E.A. (2015) Occipital Nerve Stimulation for Chronic Migraine: A Randomized Trial on Subthreshold Stimulation. Cephalalgia, 73-78. http://dx.doi.org/10.1177/0333102414534082

[15] Kember, G.E.A. (2014) Vagal Nerve Stimulation Therapy: What Is Being Stimulated? Plos One, e114498. http://dx.doi.org/10.1371/journal.pone.0114498 